\title{
Solute-solvent pair distribution functions in highly asymmetric additive hard sphere mixtures
}

Douglas Henderson

Kwong-Yu Chan

Follow this and additional works at: https://scholarsarchive.byu.edu/facpub

Part of the Biochemistry Commons, and the Chemistry Commons

\section{Original Publication Citation}

Henderson, Douglas and Kwong Y. Chan."Solute-solvent pair distribution functions in highly asymmetric additive hard sphere mixtures." The Journal of Chemical Physics 18 (1998):

9946-9947.

\section{BYU ScholarsArchive Citation}

Henderson, Douglas and Chan, Kwong-Yu, "Solute-solvent pair distribution functions in highly asymmetric additive hard sphere mixtures" (1998). Faculty Publications. 645.

https://scholarsarchive.byu.edu/facpub/645 


\title{
LETTERS TO THE EDITOR
}

The Letters to the Editor section is divided into three categories entitled Notes, Comments, and Errata. Letters to the Editor are limited to one and three-fourths journal pages as described in the Announcement in the 1 January 1998 issue.

\section{NOTES}

\section{Solute-solvent pair distribution functions in highly asymmetric additive hard sphere mixtures}

\author{
Douglas Henderson \\ Department of Chemistry and Biochemistry, Brigham Young University, Provo, Utah 84602-5700 \\ Kwong-Yu Chan \\ Department of Chemistry, The University of Hong Kong, Pokfulam Road, Hong Kong
}

(Received 23 December 1997; accepted 9 March 1998)

[S0021-9606(98)50123-7]

Of late, there has been great interest, including speculations about fluid phase equilibria, in hard sphere mixtures, especially in the region where the concentration of the large spheres (the solute) is small. ${ }^{1-11}$ Such mixtures are a simple model for colloidal suspensions. ${ }^{12,13}$

Recently, we have proposed formulas for the contact values of the radial distribution functions, and the resulting equation of state, of such a mixture. ${ }^{2,3}$ The accuracy of our formulas is supported by Monte Carlo simulations ${ }^{3}$ and by highly accurate second order integral equations. ${ }^{4,5}$ These formulas yield the exact second and third virial coefficients and yield values ${ }^{6}$ for the fourth and fifth virial coefficients that are in close agreement with the exact values of Saija et al. ${ }^{7}$ for the fourth virial coefficient and the exact values of Enciso et $a l .{ }^{8}$ for the fifth virial coefficient. It has been pointed out ${ }^{9}$ that the values of Saija et al. for the fifth virial coefficient, which differ from those of Enciso et al., are in error because of a misprint in a paper of Borstnik. ${ }^{10}$ Our formulas are the same as the well known formulas of Boublik and Mansoori et al. (BMCSL),${ }^{14}$ as extended to the contact values of the pair distribution functions by Grundke and Henderson, ${ }^{15}$ for most of the concentration range, but differ significantly when the concentration of the large spheres is small. Our formula for the solvent-solvent contact value is the same as BMCSL. The solute-solvent contact value is similar to the BMCSL but the well known ${ }^{16}$ contact value theorem, where the limit of this contact value at extreme dilution of exceedingly large particles is equal to $p V / N k T$, is grafted on to the BMCSL result, which does not satisfy this contact value theorem. To this point, our formulas are reasonably well-founded. In contrast, our expression for the solute-solute contact value, which is the most interesting of the three functions, is obtained by pure intuition. Originally, because of the ad hoc nature of our development of the latter term, we called our formulas the ad hoc formulas. However, in view of the broad range of tests that our formulas have satisfied, the original name seems inappropriate. For want of an alternative, we call our formulas the Henderson-Chan (HC) formulas.

In a recent paper, Matyushov and Ladanyi (ML) ${ }^{11}$ have performed Monte Carlo (MC) simulations of the solutesolvent pair distribution function for hard sphere mixtures where the ratio of diameters is as large as 3.5. Their system consists of one large solute sphere in a solvent of small spheres. By so doing they are able to achieve very small concentrations of the large spheres $\left(x_{2}\right.$ as small as 0.002). However, with only one large sphere, they are unable to study the solute-solute distribution function. Their simulations are complementary to our own, ${ }^{3}$ where we used 50 large spheres. As a result, we did not simulate concentrations below 0.01 . In our studies, we considered diameter ratios as large as 20 .

In addition, ML proposed a formula for the solutesolvent contact value and tested their formula and what they thought to be our formula. They found that what they thought to be our result was inferior. Regrettably, because of a misprint in one of our earlier papers, they did not use our expression. The correct formulas are found in Yau et al. ${ }^{3}$ For the convenience of the reader, we give the correct expression for $g_{12}\left(d_{12}\right)$,

$$
\begin{aligned}
g_{12}\left(d_{12}\right)= & g_{12}^{\mathrm{BMCSL}}\left(d_{12}\right)+\frac{(\delta \eta)^{2}}{(1-\eta)^{3}} \frac{d^{2}-1}{(d+1)^{2}} \\
& -\frac{(\delta \eta)^{3}}{(1-\eta)^{3}} \frac{d^{3}-1}{(d+1)^{3}} .
\end{aligned}
$$

The notation of ML has been used. This differs from the result used by ML, their Eq. (20), in the powers of the second term on the right-hand side (rhs). Equation (1) satisfies 


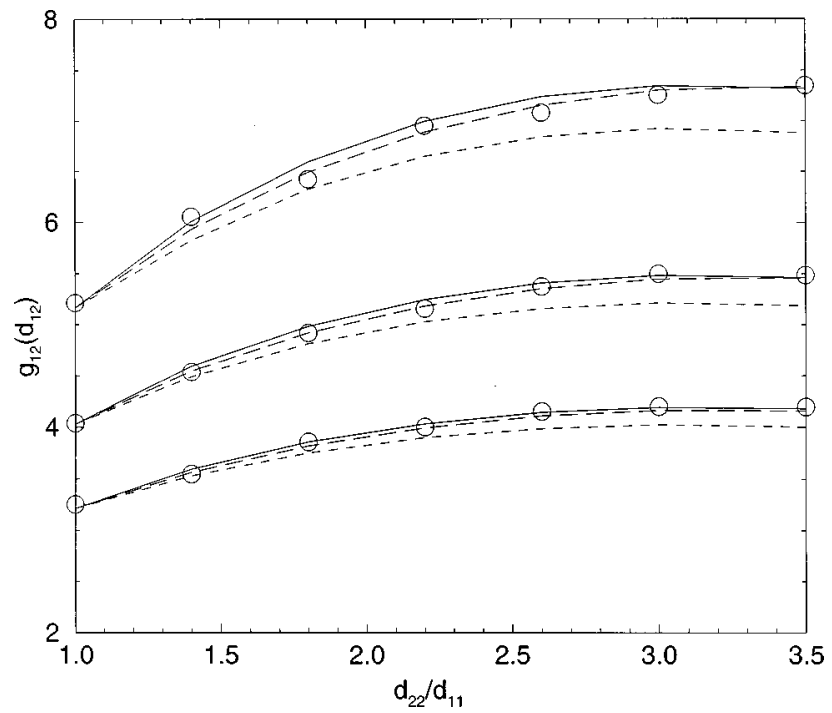

FIG. 1. Solute-solvent contact values as a function of the ratio of diameters for $x_{2}=0.0039$. The three sets of curves are, from the bottom, for $\rho^{*}$, as defined by ML, equal to $0.7,0.8$, and 0.9 . The circles are the simulation values of ML. The solid, long, and short dashed lines give the values from our expression, that of ML, and that of BMCSL, respectively. Species 2 is the large sphere.

the contact value theorem only with the correct powers. All the numerical results and graphs that are reported in our papers are correct.

In Fig. 1, we compare the results obtained from the correct version of our expression with their MC results. Their approximation and ours are virtually identical and are very good.

Since our MC results include diameter ratios as large as 20 , it is useful to compare our formula and that of ML for $g_{12}\left(d_{12}\right)$ with these results. This comparison is given in Fig. 2. In general, the two formulas give similar results. However,

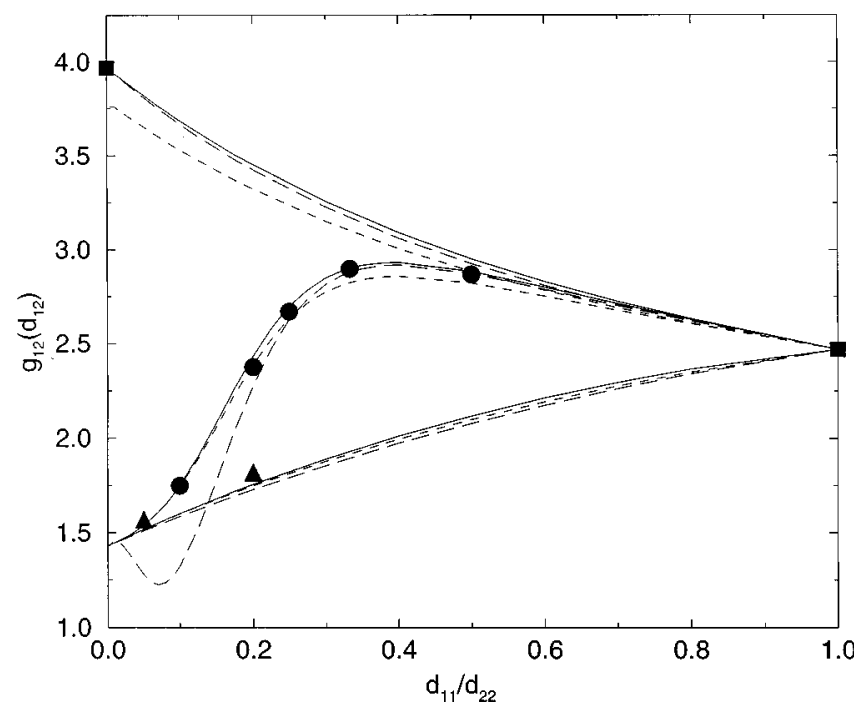

FIG. 2. Solute-solvent contact values as a function of the inverse ratio of diameters for a packing fraction of 0.3 . The solid circles and triangles give the simulation values of Yau et al. (Refs. 2 and 3) for $x_{2}=0.01$ and near 1. The squares are calculated from thermodynamic data. The solid, long, and short dashed curves give the values from our expression, that of ML, and that of BMCSL, respectively. In order from the bottom, the three sets of curves give the values of $g_{12}\left(d_{12}\right)$ for $x_{2}=1,0.01$, and 0 . Species 2 is the large sphere. for small, but not zero, $x_{2}$, the ML formula has a minimum that is not seen in the MC results. The ML formula was derived for $x_{2}=0$. There does not seem to be a problem with their fundamental formula, Eq. (16) of their paper. However, the extension to nonzero vaues of $x_{2}$, Eq. (19) of their paper, seems in need of revision. This is a small part of an admirable paper. Further, we note that this conclusion is based on a small number of simulation points. Additional simulations for large $d_{22} / d_{11}$ and small $x_{2}$ as well as at higher densities, would be valuable.

In their paper, ML state that an expression that is more accurate than the BMCSL for the entire concentration range does not yet exist. Certainly there is a need for such an expression. Respectfully, we offer, at least for the present, our formulas for this purpose. They have satisfied an impressive range of tests. In addition, they predict phase equilibria in hard sphere mixtures. ${ }^{17}$ We believe that this is the only analytic expression to do so. However, this is a rapidly developing field. We recognize that we, or some others, may propose improvements to our formulas. We welcome the prospect.

The authors are grateful to Branka Ladanyi for drawing their attention to her work with Matyushov. The financial support of the National Science Foundation (Grant Nos. CTS94-023584 and CHE96-01971) and the donors of the Petroleum Research Fund, administered by the American Chemical Society (Grant No. ACS-PRF 31573-AC9), are acknowledged with thanks. D.H. is a John Simon Guggenheim Memorial Foundation Fellow and is pleased to acknowledge this support.

${ }^{1}$ P. Fries and J. P. Hansen, Mol. Phys. 48, 891 (1983); G. Jackson, J. S. Rowlinson, and F. van Swol, J. Phys. Chem. 91, 4907 (1987); W. G. T. Kranendonk and D. Frenkel, J. Phys.: Condens. Matter 1, 7735 (1991); P. Bartlett, R. H. Ottewill, and P. N. Pusey, J. Chem. Phys. 93, 1299 (1990); Phys. Rev. Lett. 68, 3801 (1992); M. D. Madden, P. N. Pusey, and P. Bartlett, Mol. Phys. 84, 295 (1995); M. Barǒsová, A. Malijevský, S. Labík, and W. R. Smith, ibid. 87, 423 (1996); A. Malijevský, M. Barǒsová, and W. R. Smith, ibid. 91, 65 (1997); T. Coussaert and M. Baus, Phys. Rev. Lett. 79, 1881 (1997).

${ }^{2}$ D. Henderson, A. Malijevský, S. Labík, and K. Y. Chan, Mol. Phys. 87, 273 (1996); D. H. L. Yau, K. Y. Chan, and D. Henderson, ibid. 88, 1237 (1996).

${ }^{3}$ D. H. L. Yau, K.-Y. Chan, and D. Henderson, Mol. Phys. 91, 1137 (1997).

${ }^{4}$ D. Henderson, S. Sokołowski, and D. Wasan, J. Stat. Phys. 89, 233 (1997).

${ }^{5}$ D. Henderson, S. Sokołowski, and D. Wasan, Mol. Phys. 93, 295 (1998).

${ }^{6}$ D. Henderson and K.-Y. Chan, Mol. Phys. (in press).

${ }^{7}$ F. Saija, G. Fiumara, and P. V. Giaquinta, Mol. Phys. 87, 991 (1996); 89, 1181 (1997).

${ }^{8}$ E. Enciso, N. G. Almarza, D. S. Calzas, and M. A. Gonzalez, Mol. Phys. 92, 173 (1997).

${ }^{9}$ R. J. Wheatley, Mol. Phys. 93, 675 (1998).

${ }^{10}$ B. Borstnik, Vestn. Slov. Kem. Drus. 39, 145 (1992).

${ }^{11}$ D. V. Matyushov and B. Ladanyi, J. Chem. Phys. 107, 5815 (1997).

${ }^{12}$ D. Henderson and M. Lozada-Cassou, J. Colloid Interface Sci. 114, 180 (1986).

${ }^{13}$ J. N. Israelachvili, Intermolecular and Surface Forces, 2nd ed. (Academic, London, 1992).

${ }^{14}$ T. Boublik, J. Chem. Phys. 53, 471 (1970); G. A. Mansoori, N. F. Carnahan, K. E. Starling, and T. W. Leland, Jr., J. Chem. Phys. 54, 1523 (1971).

${ }^{15}$ E. W. Grundke and D. Henderson, Mol. Phys. 24, 269 (1972).

${ }^{16}$ D. Henderson, F. F. Abraham, and J. A. Barker, Mol. Phys. 31, 1291 (1976).

${ }^{17}$ D. Henderson, D. Boda, K.-Y. Chan, and D. T. Wasan, Mol. Phys. (in press). 